${ }^{2}$ Oxford Parkinson's Disease Centre University of Oxford, Oxford, UK ${ }^{3}$ School of Biosciences, College of Health and Life Sciences, Aston University, Birmingham, UK

${ }^{4}$ Department of Psychiatry and Behavioral Sciences, University of Washington School of Medicine, Seattle, WA, USA

${ }^{5}$ Department of Neurology. University of Washington School of Medicine Seattle, WA, USA

${ }^{6}$ VISN 20 Mental IIIness Research, Education and Clinical Center, VA Puget Sound Health Care System, Seattle, WA, USA.

凶e-mail:mootaz.salman@dpag.ox.ac.uk; p.kitchen1@aston.ac.uk; jiliff@uw.edu. r.m.bill@aston.ac.uk

https://doi.org/10.1038/s41583-021-00514-z

1. MacAulay, N. Molecular mechanisms of brain water transport. Nat. Rev. Neurosci. 22, 326-344 (2021).

2. Alshuhri, M. S., Gallagher, L., Work, L. M. \& Holmes, W. M. Direct imaging of glymphatic transport using $\mathrm{H}_{2}{ }^{17} \mathrm{O}$ MRI. JCI Insight 6, e141159 (2021).

Mestre, $\mathrm{H}$. et al. Cerebrospinal fluid influx drives acute ischemic tissue swelling. Science 367 eaax7171 (2020).
4. Mestre, $\mathrm{H}$. et al. Aquaporin-4-dependent glymphatic solute transport in the rodent brain elife 7 , e40070 (2018).

5. Smith, A. J., Yao, X., Dix, J. A., Jin, B.-J. \& Verkman, A. S. Test of the 'glymphatic' hypothesis demonstrates diffusive and aquaporin-4-independent solute transport in rodent brain parenchyma. eLife 6, e27679 (2017).

6. Kitchen, P. et al. Targeting aquaporin-4 subcellular localization to treat central nervous system edema. Cell 181, 784-799 (2020).

7. Ciappelloni, S. et al. Aquaporin-4 surface trafficking regulates astrocytic process motility and synaptic activity in health and autoimmune disease. Cell Rep. 27, 3860-3872 (2019)

8. Lisjak, M., Potokar, M., Rituper, B., Jorgačevski, J. $\&$ Zorec, R. AQP4e-based orthogonal arrays regulate rapid cell volume changes in astrocytes. J. Neurosci. 37, 10748-10756 (2017).

9. Yang, B., Zhang, H. \& Verkman, A. S. Lack of aquaporin-4 water transport inhibition by antiepileptics and arylsulfonamides. Bioorg. Med. Chem. 16, 7489-7493 (2008).

Competing interests

The authors declare no competing interests.

\section{Peer review information}

Nature Reviews Neuroscience thanks R. Keep and the other, anonymous, reviewer(s) for their contribution to the peer review of this work.

\title{
Reply to 'Aquaporin 4 and glymphatic flow have central roles in brain fluid homeostasis'
}

\section{Nanna MacAulay (D)}

I thank Mootaz Salman, Philip Kitchen, Jeffrey Iliff and Roslyn Bill for their additions to my recent Review (Molecular mechanisms of brain water transport. Nat. Rev. Neurosci. 22, 326-344 (2021)) $)^{1}$. In their correspondence, the authors argue that glymphatic flow deserves more attention (Aquaporin 4 and glymphatic flow have central roles in brain fluid homeostasis. Nat Rev. Neurosci. https:// doi.org/10.1038/s41583-021-00514-z $(2021))^{2}$. This topic has been reviewed extensively by researchers both in favour of $^{3}$ and in opposition to ${ }^{4,5}$ the concept (see the Review ${ }^{1}$ and correspondence for additional references). The concept was considered outside the scope of the present Review as it relates to paracellular fluid transport, as pointed out by the authors of the correspondence, rather than transmembrane water flow, which is the focus of my article (as specified in the opening pages of the Review). I therefore, again, refer the interested reader to the many reviews published on the topic. However, here, I briefly address the proposed role of AQP4 in this system in a reply to Salman and colleagues.

AQP4 has been implicated in glymphatic flow, as mentioned in the Review and highlighted by the authors of the correspondence.
Although AQP4 has been assigned a role in the paracellular flow of fluorescent probes by their reduced penetration from cerebrospinal fluid into Aqp $4^{-1-}$, Snat $1^{-1-}$ and $m d x$ mouse brains (see the correspondence for references), the mechanism by which cellular AQP4 could contribute to paracellular fluorescent tracer movement remains incompletely, if at all, understood. In the original publication by Iliff et al. ${ }^{6}$, in which the glymphatic system was coined, the authors proposed that water would flow freely through AQP4 across the astrocytic endfoot and exit again towards the interstitium (see supplementary figure 9A and associated legend in REF. and figure 1 in the correspondence ${ }^{2}$ ). The fluorescent tracer would then "follow the resulting osmotic gradient" between the paravascular space and the interstitium and thus represent glymphatic flow ${ }^{6}$. However beautiful such a strategy may be, it is challenging to align it with basic biophysical considerations. First, pressure-dependent AQP4-mediated water entry into the astrocyte would be prevented by the resulting oppositely directed osmotic gradient. Second, the proposed interstitial osmotic gradient could only arise by (the non-endfeet) AQP4 permitting water transport against an osmotic gradient, which it does not. Third, the fluorescent particles will not "follow the resulting osmotic gradient into the interstitium through intercellular clefts" ${ }^{36}$ as water follows particles by osmosis, not the opposite. Last, osmosis occurs across a semipermeable membrane and not through intercellular clefts.

To my knowledge, AQP4-dependent water transport through AQP4 has not been documented in the glymphatic hypothesis, which, however, does not prevent AQP4 from serving a structural role in the system. Notably, Aqp $4^{-/-}$mice display severely reduced expression of protein anchoring complexes in the astrocytic endfeet ${ }^{7}$, which may affect endfoot polarization of other astrocytic membrane proteins and thereby indirectly influence astrocyte function in a manner that could affect the paracellular flow of fluorescent particles. A specific and efficient inhibitor of AQP4 would provide the tool to reveal a requirement for AQP4-dependent transcellular water flux to support paracellular flow of fluorescent molecules. The authors rightly point out the futile search for such inhibitors. However, TGN-020 is among the most promising of its kind: it displays near-absent binding to $A q p 4^{-/-}$mouse brain tissue ${ }^{8}$, it causes reduced rodent brain oedema formation ${ }^{9}$ and it acts directly on the pore of AQP4 in an isoform-specific manner ${ }^{10}$. I highly welcome future determination of direct versus indirect roles of AQP4 in a glymphatic system, as well as in its pathological relocalization promoting oedema formation ${ }^{11}$, and anticipate the associated delineation of the underlying driving forces supporting the proposed AQP4-dependent transmembrane water flow. It will be my pleasure to include such mechanistic findings in future review articles on the molecular mechanisms of brain water transport.

\section{Nanna MacAulay iD Department of Neuroscience, Faculty of Health and Medical Sciences, University of Copenhagen Copenhagen, Denmark e-mail:macaulay@sund.ku.dk https://doi.org/10.1038/s41583-021-00515-y}

MacAulay, N. Molecular mechanisms of brain water transport. Nat. Rev. Neurosci. 22, 326-344 (2021). 2. Salman, M. M., Kitchen, P., Iliff, J. J. \& Bill, R. M. Aquaporin 4 and glymphatic flow have central roles in brain fluid homeostasis. Nat. Rev. Neurosci. https:// doi.org/10.1038/s41583-021-00514-z (2021).

3. Mestre, H., Mori, Y. \& Nedergaard, M. The brain's glymphatic system: current controversies. Trends Neurosci. 43, 458-466 (2020).

4. Smith, A. J. \& Verkman, A. S. The "glymphatic" mechanism for solute clearance in Alzheimer's disease: game changer or unproven speculation? FASEB J. 32 , 543-551 (2018).

5. Abbott, N. J., Pizzo, M. E., Preston, J. E., Janigro, D. \& Thorne, R. G. The role of brain barriers in fluid movement in the CNS: is there a 'glymphatic' system? Acta Neuropathol. 135, 387-407 (2018).

6. Iliff, J. J. et al. A paravascular pathway facilitates CSF flow through the brain parenchyma and the clearance 


\section{CORRESPONDENCE}

of interstitial solutes, including amyloid $\beta$. Sci. Trans / Med. 4, 147ra111 (2012).

7. Eilert-Olsen, M. et al. Deletion of aquaporin-4 changes the perivascular glial protein scaffold without disrupting the brain endothelial barrier. Glia 60 , 432-440 (2012)

8. Nakamura, Y. et al. Development of a novel ligand $\left[{ }^{11} \mathrm{C}\right] \mathrm{TGN}-020$, for aquaporin 4 positron emission tomography imaging. ACS Chem. Neurosci. 2, 568-571 (2011).
9. Igarashi, H., Huber, V. J., Tsujita, M. \& Nakada, T. Pretreatment with a novel aquaporin 4 inhibitor, TGN-020, significantly reduces ischemic cerebral edema. Neurol. Sci. 32, 113-116 (2011).

10. Toft-Bertelsen, T. L et al. Clearance of activityevoked $\mathrm{K}^{+}$transients and associated glia cell swelling occur independently of AQP4: a study with an isoform-selective AQP4 inhibitor. Glia 69 28-41 (2021)
11. Kitchen, P. et al. Targeting aquaporin-4 subcellular localization to treat central nervous system edema. Cell 181, 784-799 (2020).

\section{Competing interests}

The author declares no competing interests.

\section{Peer review information}

Nature Reviews Neuroscience thanks R. Keep and the other, anonymous, reviewer(s) for their contribution to the peer review of this work. 